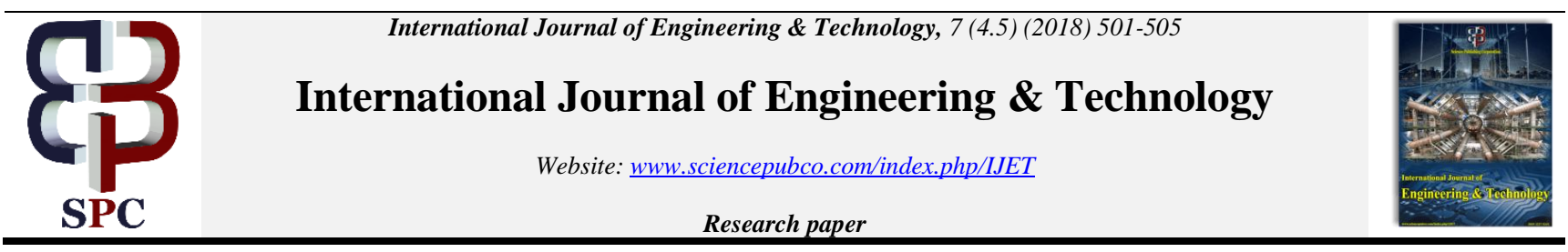

\title{
Analysis and tracking of animal movements through granulation of temporal domain (GTD)
}

\author{
Neelam Rawat $^{1}$ *, J. S. Sodhi ${ }^{2}$, Rajesh K. Tyagi ${ }^{3}$ \\ ${ }^{1}$ KIET Group of Institutions \\ ${ }^{2}$ Amity University \\ ${ }^{3}$ SRM University \\ *Corresponding author E-mail:
}

\begin{abstract}
Thanks to technological advancement in tracking systems, observing the moving patterns of a population is easier now. Capturing the movement patterns is mostly done through various tracking technologies like GPS, GIS etc. but these tracking systems basically require one or more combination of the same, and also, a node or tag to be placed on the moving object. Tracking of movement patterns basically employs a motion model which describes how the image of the object might change in different possible positions of the object during video surveillance. To track multiple objects without deformation during movement, the granulation of temporal domain (GTD) is proposed.

GTD method is for demodulation of granule adaptation of video image segmentation on the basis of granular computing methodology. In image segmentation, i.e., image granulation through temporal domain, it improves the quality of surveillance important for wild-life protection, and decision making in terms of security.
\end{abstract}

Keywords: Video Surveillance; Image Granulation; Background Subtraction; Granular Computing; Temporal and Spatial Domains.

\section{Introduction}

While detecting objects [2], [19], the proposed model tracks a single-target object continuously, and accurately measures the distance moved by the target. The objective of single-target tracking is to associate targeted object to the consecutive video frames identified by the video- frame sequences [17]. Real-time processing is required to track the object successfully and effectively, in order to further increase its quali- ty and reduce the quntity of data to be either stored or transferred.

Our work's aim is to detect moving objects which are in the foreground and through the pixels from motion and time-duration, define these objects [7-8]. Our algorithm segments group of pixels consistently according to motion points. These motion points correspond to moving targeted objects [9-10] that may further process from the projection of those moving points and its neighborhoods [11].

The algorithmic approach has been discussed in section 2 of the operational work in methodology. Firstly, the motion of camera is comput- ed so that images can be rectified in step 1 , which is finally converted into frames. Background detachment will be inclusive in the flow of the algorithm. And comparison of various frames will be in the matrix form individually. Section 3 elaborates the respective

Framework for the proposed algorithm. Section 4 shows the granular technique concept, image granulation, followed by object granulation in Section 5, with effective results in Section 7. The object in the image streams is finally tracked for further processing, analysis of motion and movement.

\section{Methodology}

Operational work: While executing the program, a video camera records the area where animals are the objects for input video to detect the movement. By RGB2GRAY, a pre-defined proce- dure that converts colorful video to gray. Then, frames digitize the gray level video output, i.e., the software analyzes individual frames from various background-subtraction algo- rithms so that objects are easily tracked from the background [3], [19]. The proposed method creates motion image from con- secutive pair of frames. For this, moving object extracts from frame 1 , as at frame 0 there is no reference image [4]. Through this continual process, motion would be estimated based on previous image. Then transformation matrix will be aligned and both the images will be processed. The whole program will be repeated until the animal schedule on the basis of time domain may not be found. Finally, the result will be stored, observed and compared. The whole methodology automatically constructs a background model through a time domain for effective detec- tion, and finally frames a temporal schedule for those animals. Here, the image matrix, which is, generated (algorithm step 12-

13) To maintain the correlation of objects over time until each moving object is detected and analyzed. The overall architecture is shown in Fig. 1.

\subsection{Algorithm}

Step 1: Open/ Read the video file.

Step 2: Divide the video file into frames and count the number of frames (say $n$ ). 
Step 3: For (i $=1$ to $n)$

Read the frame one by one and store into an array of record (say r).

Step 4: Convert each frame into an image.

Step 5: Convert a series of filename with .bmp extensions.

Step 6: Store each frame into files

Step 7: Background would be given as bkground.bmp.

Step 8: Now separate moving object from individual frames.

Step 9: For ( $\mathrm{i}=1$ to $\mathrm{n})$

Mobject[i] =filename[i].bmp-bkground.bmp.

Step 10: Now create a matrix of moving object after comparing each one.

Step 11: For (i= 1 to $n-1)$

For $(\mathrm{j}=\mathrm{i}+1$ ton $)$ Motion[i] [j] = Obj_Cmp(Mobject[i], Mobject[j])

Step 12: Repeat entire process for each day at the same time interval $\mathrm{t}$-to- $\mathrm{t}+1$ and store the result in another mo- tion matrix

Step 13: Two comparing function

Mot_Row_Compare (entire rwo) and Mot_Column_Compare(entire column) for analyz- ing the motion of an object.

\section{Proposed architecture for image granulation}

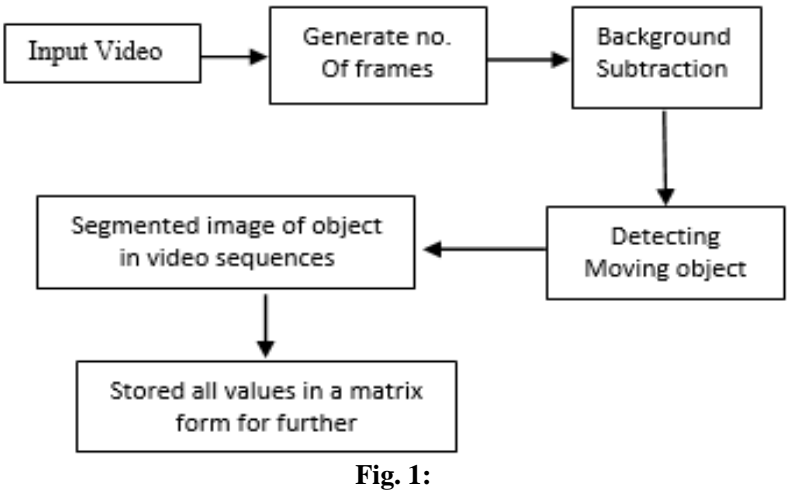

The architectural model shows the granulation of an image from a video sequence. To identify the moving objects from that part of video frame, we simply use the basic approach of background subtraction. Through the entire process, the most challenging part is to develop an effective background subtraction algorithm, so that, during the image illumination, it should be robust. To resolve this, the model classifies the image pixels on the basis of granules, and then groups them together according to their schedule, in order to identify object time domain. Generally, background segmentation techniques result into some bitmap image, where 0 is considered to be the background and 1 to be foreground. Merging these bitmaps is actually called the process of object extraction. An image matrix is generated from these consecutive image frames, and a counter advances whenever movement is detected at a particular location with respect to time domain in a frame. Finally, the method proceeds until the image granulation of temporal domain observes the moving object to make it ready for further analysis and observation.

\section{Concept of image granulation through rough sets}

Let us consider an information system, say $\mathrm{P} \leq \mathrm{Z} 1$, where $\mathrm{Z} 1$ represents the universe and $P$, set of attributes [12].

Suppose $\mathrm{Q} \subseteq \mathrm{P}$, then the set $\{\mathrm{z} \& \mathrm{Z} 1:|\mathrm{z}| \mathrm{Q} \subseteq \mathrm{Z} 2\}$ would be $\mathrm{Q}$-lower of $Z 2$ i.e., $Q Z 2$ which will always be the subset of Z2. Similarly, the set $\{\mathrm{z} \& \mathrm{Z} 1:|\mathrm{z}| \mathrm{Q} \neq \Phi\}$ represents $\mathrm{Q}$-upper of $\mathrm{Z} 2$ i.e., QZ2 that indicates a non-zero interaction with $\mathrm{Z} 2$.

Here, $|z| Q$ represents equivalence class of object $z \& Z 1$. Roughness of any set Z2 with respect to $\mathrm{Q}$ will be

Rimage $=$ I $-($ QZ2 / QZ2)
If the universe, $\mathrm{Z} 1$ of any image I of size AXB consists of pixels, then subsets of $\mathrm{Z} 2$ would be over-lapping window images of unequal size. Each window would be considered as a granule of that image which is further approximated by rough sets depending upon the granulation process [1]. To detect the object, we need to form granules by plotting the pixels of an image on gray-level similarity as of limited discernibility. Figure 2 illustrates the set of granules with upper and lower approxima- tions of the pixels of an image. In this approach, granular sets applied to various areas of image processing rather than on video processing. Here, our focus is on granule sets. Granular Computing Approach simply handles ambiguities and uncertainties of video images and show effective results. Unlike the conventional methodologies which deals with object tracking from previous frames and consumes large memory, our approach requires less memory, as only in- formation is stored in matrix form from the previous two frames

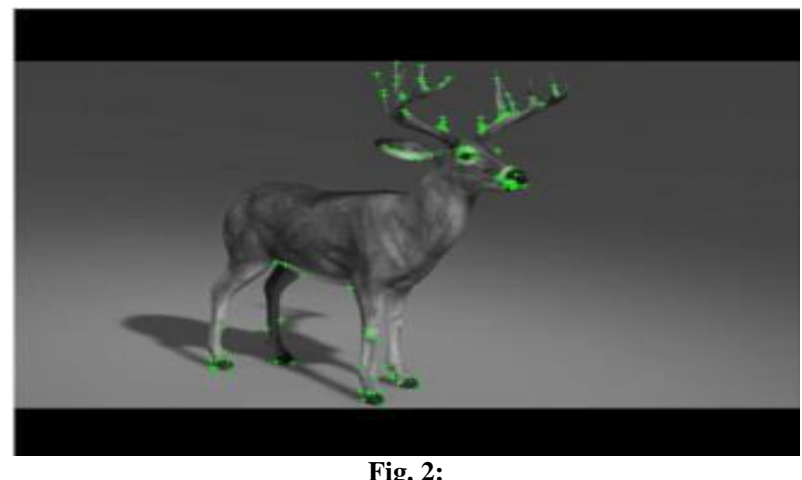

Fig. 2:

\section{Object tracking and granulation}

The initial step is to find the object from a video sequence as a compound granule in the frame. The object can be protruding from its background on the basis of its spatial and temporal like- liness. In this section, we show how the proposed approach di- vides spatial and temporal data for reconstructing the object movement from the targeted frame in the whole tracking pro- cess. Through ths intersection of both the data, may get the re- gion of the object which may common to both [13][14]. The discernment of an image from moving object depends on its effective granulation. To segment any image, object granulation can be a useful tool [16][18]. The basis notion underlying the granular sets from object granulation is to contrive extent to which a given set of objects approximates another object of interest [14][20]. Object granulation could be of different types with granules of equal or unequal size on the basis of application, even though unequal granules would be more genuine to the real life world. From this point of state, we need to change the granule parameters (either equal or unequal size) to clandes- tine the object pixels with the smallest granule. So we measure each parameterized granule as:

$\mathrm{T}(\mathrm{n}(\mathrm{p}, \mathrm{q}, \mathrm{r}, \mathrm{s}))=\frac{o 1}{01+o 2}$

Where $\mathrm{O} 1$ is the total object pixels within each granule, and $\mathrm{O} 2$ is the total number of pixels in the image background.

\section{Results and discussion}

The results obtained from object tracking estimations through granulation of time domain (GTD) are the non-deterministic qualities. The image patterns from video sequences are shown in Figure 3, where large samplings of pixels are not experimented to obtain granulated images. After converting granulated imag- es into frames, we get two sets of images shown in Figure 4(a) and 4(b), respectively, that show motion images from consecu- tive frames. As foresaid, each moving object image would be the difference between the two consecutive frames in the input vid- eo for detecting 
movement. The suggested approach for detec- tion and tracking of objects are implemented and examined on various image sequences. For illustration of results, we are con- sidering two video sequences and 4-5 frames from each se-

quence in Figure 5, which fuel complete picture

of proposed methodology. The object is shown in Figure 6 with the detailed dimensions of the image reference point of pixels. From the results shown, much more effective results of tracked and detected objects are found.
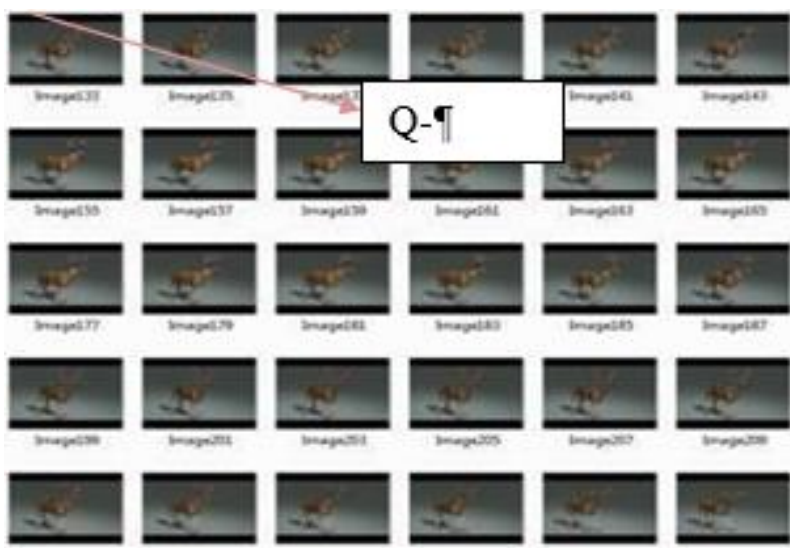

Fig. 3:

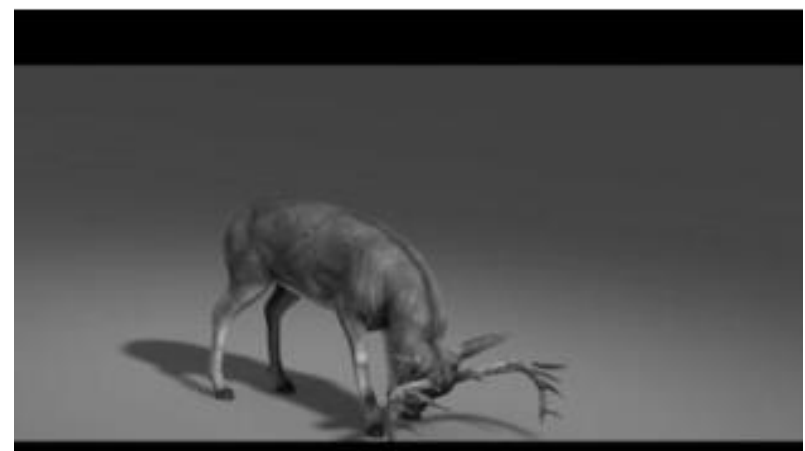

Fig. 4: a.
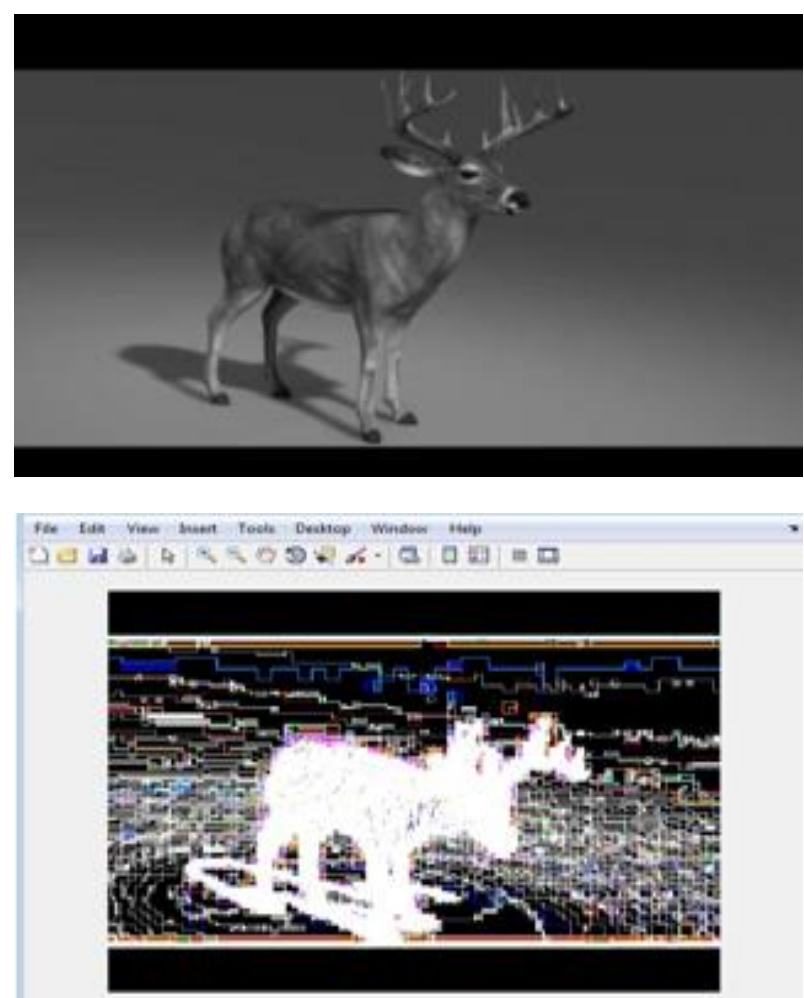

Fig. 4: B.

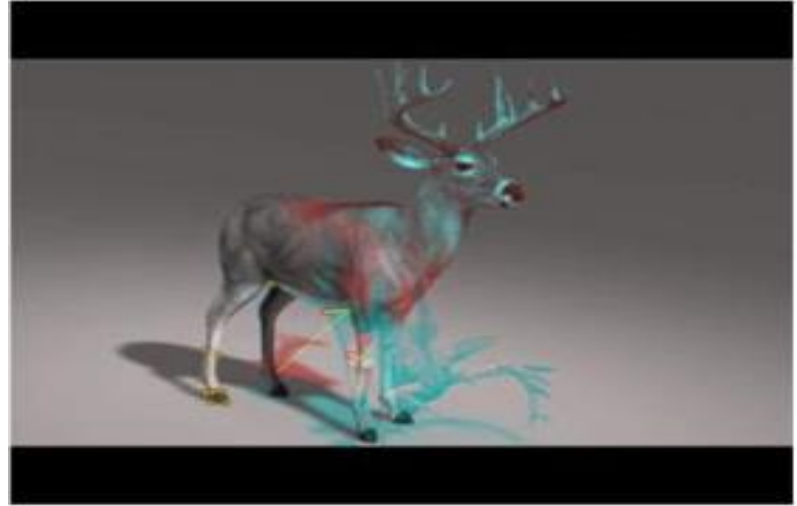

Fig. 5:
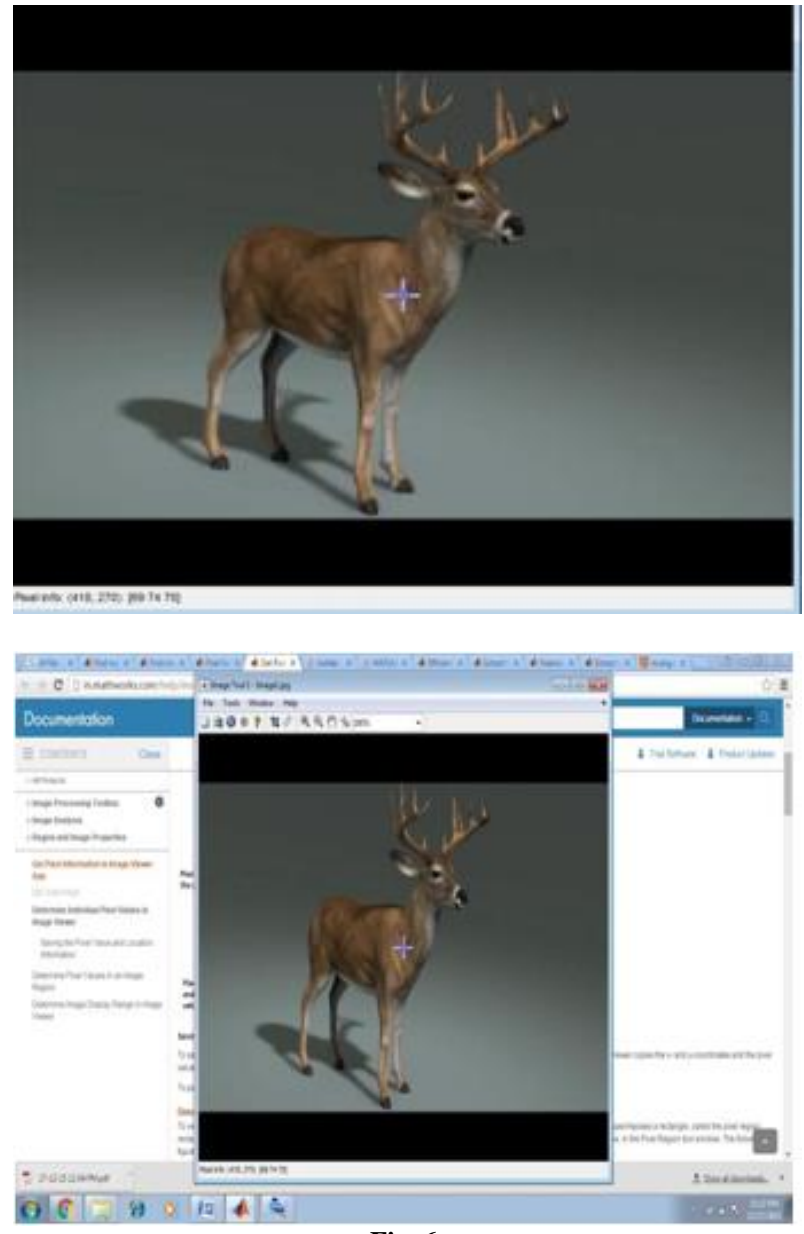

Fig. 6:

\section{Aim, application and conclusion}

Two situations are encountered while detecting and tracking of objects independently from the background as:

- $\quad$-Stationary Cameras (fixed viewpoint)

- $\quad$-Moving Cameras (moving viewpoint)

Object motion can be measured by change in speed vector of an object and object movement by sensing physical movements in given areas. Broad applications of our approach could be as follows:

\section{Surveillance Applica- tions Control Applications}

- Security Cameras

- Traffic Monitoring

- Population counting
- Object Avoidance

- Automatic Guidance \& Controlling

- Head Tracking for Video Conferencing 
While using static camera, simple frame difference of two imag- es shows moving objects for comparison in reference to back- ground image. Further, comparison has been done between tar- geted or current image with reference image pixel by pixel, through which differences and similarities are identified and observed. So, this parametric value makes it less memory inten- sive. In this way, the model copes also with multi-model back- ground distributions.

But, comparatively, our approach may provide various frameworks which comparatively sound effective and useful via its applications.

Our aim is to coordinate the different frames for effective analysis and to iteratively refine them to obtain object statistics,

so granular set on the basis of time domain is finally achieved as demonstrated in Figure 8.

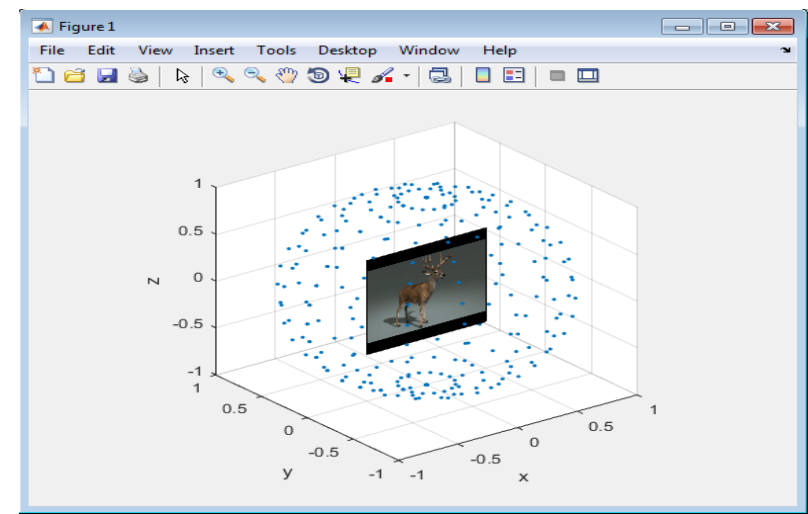

Fig. 7:
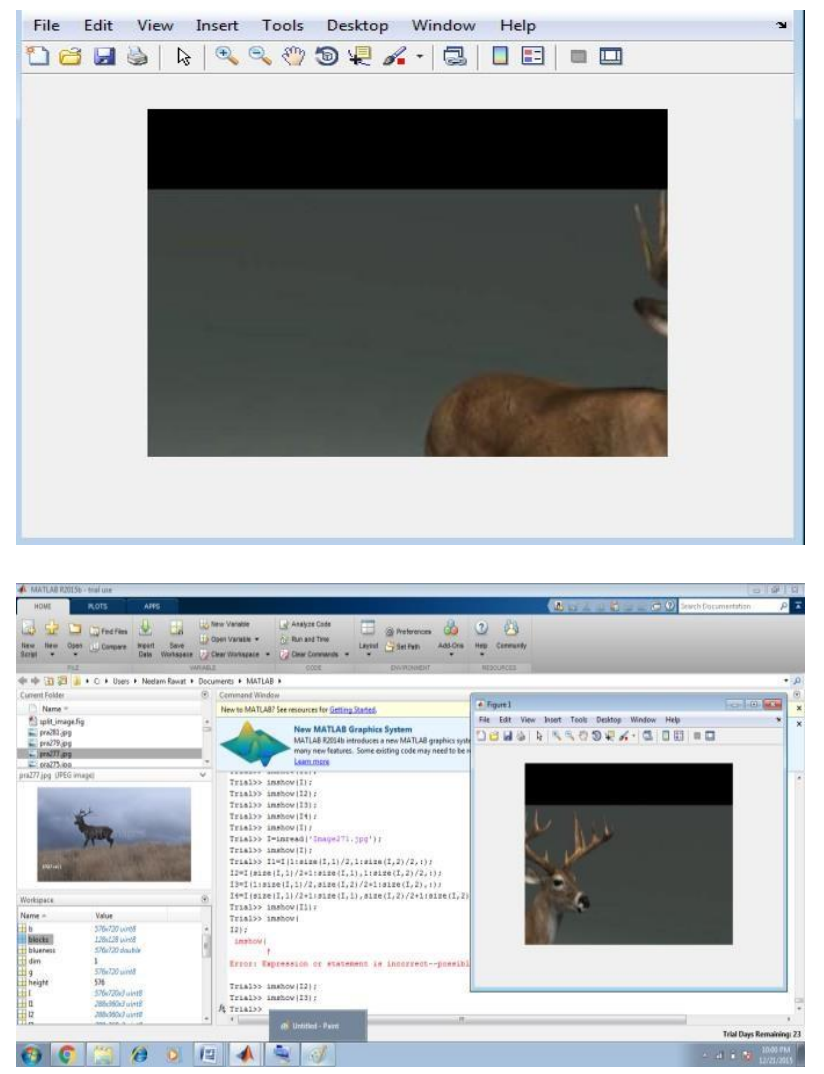

Fig. 8:

\section{Conflict of interests}

The authors declare that there is no conflict of interest's regard- ing the publication of this paper.

\section{References}

[1] Debarati Chakrabortya ET. al., "Granulation, rough entropy and spatio-temporal moving object detection", Applied Soft Computing 13 (2013) 4001-4009.

[2] Nan Lu ET. al., "An Improved Motion Detection Method for Real-Time Surveillance", IAENG International Journal of Com- puter Science, 35:1, IJCS 35_1_16.

[3] Lucas P.J.J. Noldus ET. al., "Computerised video tracking, movement analysis and behaviour recognition in insects", Com- puters and Electronics in Agriculture 35 (2002) 201227.

[4] Jianhui Zhao ET. al., "Contour Points based P2P Algorithm for Shape Matching and Image Retrieval", Appl. Math. Inf. Sci. 8, No. 1, 37-43 (2014).

[5] Aurelie Bugeau ET. al., "Detection and segmentation of moving objects in highly dynamic scenes" IEEE Conference on Computer Vision and Pattern Recognition, CVPR'07, 2007. pp 1-7, Minneapolis.

[6] S. Jeeva ET. al., "Survey on background modeling and foreground detection for real time video surveillance", 2nd Interna- tional Symposium on Big Data and Cloud Computing (ISBCC'15), Procedia Computer Science 50 ( 2015 ) $566-$ 571

[7] Luke Bermingham ET. al., "A general methodology for ndimensional trajectory clustering”, 40957- 4174/ 2015 Elsevier Ltd.

[8] Mrinali M. Bhajibhakare ET. al. "To Detect and Track Moving Object for Surveillance System”, International Journal of Innovative Research in Computer and Communication Engineering Vol. 1, Issue 4, June 2013, ISSN (Print) : 2320 9798.

[9] ISSN (Online): 2320 - 9801.

[10] Alfredo Petrosino ET. al., "Rough fuzzy set-based image compression", Elsevier, Fuzzy SetsandSystems160 (2009)1485- 1506

[11] Adnan Khashman, "Automatic Detection, Extraction and Recognition of Moving Objects", International Journal of Sys- tems APPLICATIONS, ENGINEERING \& Development Issue 1, Volume 2, 2008.

[12] Mrinali M. Bhajibhakare ET. al., "To Detect and Track Moving Object for Surveillance System", International Journal of Innovative Research in Computer and Communication Engineering Vol. 1, Issue 4, June 2013.

[13] H. Fradietal "Spatio-temporal crowd density model in a human detection and tracking framework", Signal Processing: Image Communication 31(2015)100-111 0923-5965/\& 2014 Elsevier.

[14] C. Stasch ET. al. "Meaningful spatial prediction and aggregation", Elsevier: Environmental Modelling \& Software 51 (2014) $149 \mathrm{e} 165$

[15] Miao Lin ET. al. "Mining GPS data for mobility patterns: A survey", 2013 Elsevier B.V., Pervasive and Mobile Computing 12 (2014) 1-16.

[16] David M. Curry "An Algorithm for Clustering Animals by Species Based upon Daily Movement", ScienceDirect Procedia Computer Science 36 (2014) 629 - 636

[17] Lee,M.Kang "Geospatial Big Data: Challenges and Opportunities", 2214-5796/ 2015 Elsevier, Big Data Research 2 (2015) 74-81.

[18] T.D. Pham, "Pattern recognition by active visual information processing in birds", 0020-0255/ 2014 Elsevier, Infor- mation Sciences 270 (2014) 134-142.

[19] Hongbing Liu ET. al., "Image Superresolution Reconstruction via Granular Computing Clustering", Computational Intel- ligence and Neuroscience Volume 2014, Article ID 219636/ 10.1155/2014/219636

[20] F. Dornaika ET. al., "Decremental Sparse Modeling Representative Selection for prototype selection", 0031-3203/ 2015, Pattern Recognition, Elsevier.

[21] McFarlane, N.J.B. and Schofield, C.P. (1995) 'Segmentation and tracking of piglets in images', Machine Vision and Applications, 8(3), pp. 187-193. doi: 10.1007/bf01215814.

[22] Wren, C.R., Azarbayejani, A., Darrell, T. and Pentland,

[23] A.P. (1997) 'Pfinder: Real-time tracking of the human body', IEEE Transactions on Pattern Analysis and Machine Intelligence, 19(7), pp. 780-785. doi: 10.1109/34.598236.

[24] Stauffer, C. and Grimson, W.E.L. (2000) 'Learning patterns of activity using real-time tracking', IEEE Transactions on 
Pat- tern Analysis and Machine Intelligence, 22(8), pp. 747757. doi: 10.1109/34.868677.

[25] Cucchiara, R., Grana, C., Piccardi, M. and Prati, A. (2003) 'Detecting moving objects, ghosts, and shadows in video streams, IEEE Transactions on Pattern Analysis and Machine Intelligence, 25(10),pp. 1337-1342. doi: 10.1109/tpami.2003.1233909.

[26] Zheng, Y.-J. (1995) 'Feature extraction and image segmentation using self-organizing networks', Machine Vision and Applications, $8(5), \quad$ pp. 262-274. doi: 10.1007/s001380050008.

[27] Ricquebourg, Y. and Bouthemy, P. (2000) 'Real-time tracking of moving persons by exploiting spatio-temporal image slic- es', IEEE Transactions on Pattern Analysis and Machine Intelli- gence, 22(8), pp. 797-808. doi: 10.1109/34.868682.

[28] Koutras, P. and Maragos, P. (2015) 'A perceptually based spatio-temporal computational framework for visual saliency estimation', Signal Processing: Image Communication, 38, pp. 15-31. doi: 10.1016/j.image.2015.08.004.

[29] Noldus, L.P.J.J., Spink, A.J. and Tegelenbosch, R.A.J. (2001) 'EthoVision: A versatile video tracking system for auto.

[30] mation of behavioral experiments', Behavior Research Methods, Instruments, \& Computers, 33(3), pp. 398-414. doi: 10.3758/bf03195394.

[31] Zheng, X., GAO, Z. and Luo, W. (2014) 'Feature of statistical projection algorithm-based image retrieval', Applied Math- ematics \& Information Sciences, 8(2), pp. 721-725. doi: $10.12785 / \mathrm{amis} / 080231$.

[32] Liu, T.-L. In addition, Chen, H.-T. (2004) 'Real-time tracking using trust-region methods', IEEE Transactions on Pattern Analysis and Machine Intelligence, 26(3), pp. 397-402. doi: 10.1109/tpami.2004.1262335. 